This item was submitted to Loughborough's Research Repository by the author.

Items in Figshare are protected by copyright, with all rights reserved, unless otherwise indicated.

\title{
Application of adhesives in MEMS and MOEMS assembly: a review
}

PLEASE CITE THE PUBLISHED VERSION

PUBLISHER

(C) IEEE

LICENCE

CC BY-NC-ND 4.0

REPOSITORY RECORD

Sarvar, Farhad, David A. Hutt, and David C. Whalley. 2019. "Application of Adhesives in MEMS and MOEMS Assembly: A Review”. figshare. https://hdl.handle.net/2134/3981. 
This item was submitted to Loughborough's Institutional Repository (https://dspace.lboro.ac.uk/) by the author and is made available under the following Creative Commons Licence conditions.

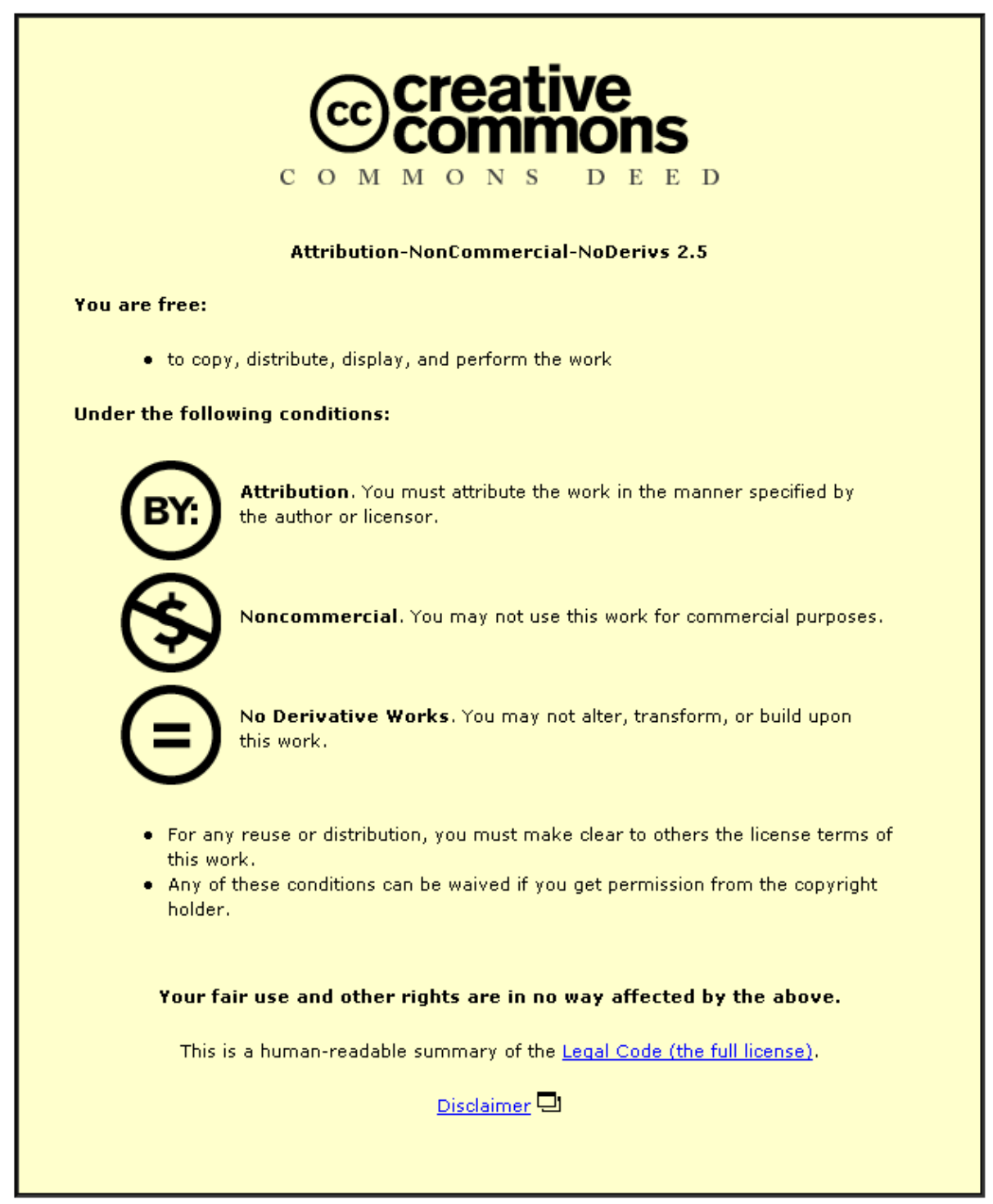

For the full text of this licence, please go to: http://creativecommons.org/licenses/by-nc-nd/2.5/ 


\title{
Application of Adhesives in MEMS and MOEMS Assembly: A Review
}

\author{
Farhad Sarvar, David A. Hutt and David C. Whalley \\ Wolfson School of Mechanical and Manufacturing Engineering, \\ Loughborough University, \\ Loughborough, Leics, LE1 13 TU, UK. \\ Phone +44-1509-227661, Fax +44-1509-227648 \\ Email: F.Sarvar@Lboro.ac.uk, D.A.Hutt@Lboro.ac.uk, D.C.Whalley@Lboro.ac.uk
}

\begin{abstract}
This paper presents a review of the recent literature on the use of adhesives in MEMS packaging applications. The aim of this review has been to establish the current applications of adhesives in MEMS and MOEMS assembly and to investigate the limitations and future requirements of these materials. The review has shown that while there is a wealth of information available on the packaging of MEMS devices, there is very limited detail available within the public domain regarding the specific uses of adhesives and in particular exactly which products are in use. The paper begins with an overview of the uses of adhesives in MEMS packaging, subdivided into sections on structural adhesives, adhesives for optical applications and other applications. The paper then describes methods for adhesive dispensing and issues with adhesive use which affect the reliability of the package. The reliability of MEMS devices assembled using adhesives is a challenging issue, being more than a simple combination of electrical, mechanical and material reliability. Many failure modes in MEMS devices can be attributed to the adhesives used in the assembly; for example, thermal expansion mismatches can cause stress in the die attach, while outgassing from epoxies can cause failure of sealed devices and contamination of optical surfaces.
\end{abstract}

\section{INTRODUCTION}

Microelectromechanical systems (MEMS) or microsystems are generally either a single micromachined device or a number of such devices and their associated control/signal processing circuits providing a defined function. Examples are pressure sensors, microactuators etc. While the electronics are fabricated using integrated circuit (IC) process sequences, the micromechanical components are fabricated using micromachining processes that selectively etch away parts of the silicon wafer or add new structural layers to form the mechanical and electromechanical devices.

To allow the integration of such devices into a complete product, they need to be packaged in some form. Figure 1 summarises the areas of the package in which adhesives might be used. Adhesives are generally used as structural layers to bond different layers together (e.g. wafer-to-wafer, or wafer-tosubstrate), sealing the device or as a protective coating (encapsulant). They can also be used for optical applications such as the mounting of fibre optics, or in the formation of microlenses in Micro-opto-electro- mechanical (MOEMS) devices [1]. Electrically conducting adhesives have been used to form electrical joints between components, for example in a flip-chip configuration. As part of this review, a number of other applications have been identified including: photoresist for lithographic patterning in MEMS and MOEMS, and microgasketing in microfluidic systems [2].

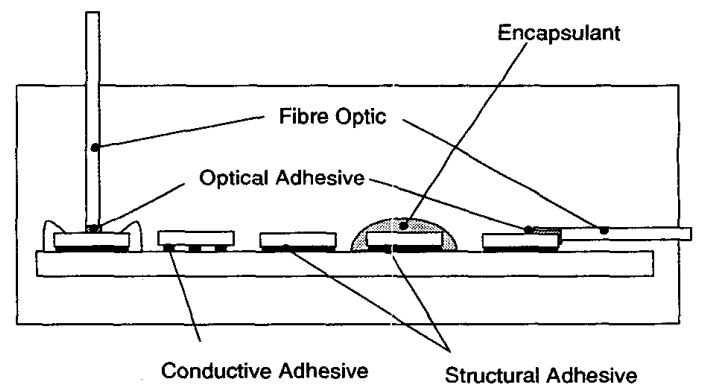

Fig. 1 Adhesive Use in Microsystems

The reliability of MEMS devices assembled using adhesives is a challenging issue, being more than a simple combination of electrical, mechanical and material reliability. Many failure modes in MEMS 
devices, inevitably impacting on reliability, can be attributed to the adhesives used in the assembly; for example, thermal expansion mismatches can cause stress in the die attach, while outgassing from epoxies can cause failure of sealed devices and contamination of optical surfaces.

The aim of this paper is to review the use of adhesives in the assembly and packaging of MEMS and MOEMS devices and to consider the failure mechanisms involving adhesives as reported by workers in the area.

\section{StruCtural AdHesive APPLiCations}

In general, structural adhesives are used to attach devices to a substrate within a package or to join together two wafers (wafer bonding) that form a complete structure. In addition, adhesives can be employed as encapsulants to seal the entire package or simply to provide a protective layer over an individual die. While many applications make use of adhesives, there is limited information in the literature regarding detailed material usage. The following sections summarise a number of example applications where structural and electrically conducting adhesives have been reported and where specific reference is made to the materials in use.

In a project report published by Nasa Glenn Research Center [3], a proof of concept has been described for the fabrication of a MEMS sensor for total pressure measurement. In this project Tra-Cast 3145 epoxy is used to cement the pressure sensor and lead wires into position. They used gold interconnect wires and the connection between the gold wires, sensor die and the lead wires were made with Epo-Tek H20E, an electrically conductive silver loaded epoxy.

Aeroprobe corporation [4] in collaboration with Texas A\&M University have developed a MEMS based, high-performance, fast-response, multi-sensor pressure probe of miniature size for velocity and pressure measurement in unsteady and turbulent flow fields. They have succeeded in fabricating an array of 5 bossed-diaphragm absolute pressure sensors $250 \mu \mathrm{m}$ square. Each of the pressure sensors is coupled with a corresponding hole in the sensor head, with no chance of leakage or cross-talk between ports. This is accomplished by sealing all of the tubes to the MEMS array in one epoxy setting, then sealing the tip to the tubes in a separate epoxy setting. They have developed a fixture, referred to as the "sealer", which is used to accurately position the tubes on the MEMS array, so that epoxy can be placed around the outside diameter of each tube. After the epoxy has cured, the tip is fitted over the ends of the tubes and new epoxy is placed around the outside of each tube before completing the joint.
Adhesives have been widely used as conformal encapsulants for many years. Reliable encapsulation materials include high purity epoxies, room temperature vulcanised (RTV) silicones and fluorinated silicone based materials. The key specifications for MEMS device encapsulants are [5]: resistance to mobile ion permeation and high humidity, constant thermal and mechanical properties within wide temperature extremes and low internal stress for guaranteeing accurate sensing. Good adhesion and solvent resistance are also of importance.

Metal loaded conductive adhesives are extensively used for attaching silicon die to packages and substrates. While solder is an attractive joining material because of its high thermal conductivity, its high melting temperature causes problems in die attach applications. Lower process temperatures are possible with die attach adhesives, but their low thermal conductivity has been a significant drawback. The benefit of these materials is their ease of processing compared to other methods such as soldering. In devices with higher heat dissipation thermal conductivity of the bond material is of greater importance. Silver filled adhesives are now available with thermal conductivities of e.g. $13 \mathrm{~W} / \mathrm{mK}$ for Thermoset, MD-140 [6] to 25W/mK for Dexter, QMI 5030 [7] compared to $2-5 \mathrm{~W} / \mathrm{mK}$ for typical conductive epoxies [8].

Heschel et al. at MIC [9] have used a stacking technology for integrated packaging of an intelligent transducer formed by a micromachined silicon transducer and an IC chip. Transducer and circuitry are stacked with an intermediate chip. The transducer and intermediate chip are bonded by flip chip solder bumping. Conductive adhesives combined with gold studs are however used for bonding the two-layer stack to the IC chip. In collaboration with Microtronic $\mathrm{A} / \mathrm{S}$ and DELTA, MIC has also used the stacking technology for the development of an intelligent microphone for hearing aids. ASIC chips and a sealing membrane are mounted on top of the chip stack using adhesive. The complete microsystem is then encapsulated in a conductive adhesive for EMI shielding.

In addition to the applications outlined above, adhesives are utilised in MEMS assembly in a number of more challenging areas:

\section{Selective Adhesive Deposition}

The assembly of MEMS often requires a waferjoining process where two micromachined structures are bonded together at a low temperature. Adhesives can offer low temperature bonding (depending on the adhesive), require no special coating of the surfaces, as long as the interfacing materials have enough binding force, and will tolerate particulate 
contamination and unevenness in the surfaces to be joined as long as their dimensions are smaller than the adhesive thickness [10].

In MEMS applications wafers to be bonded are also frequently three-dimensionally patterned, often with deep grooves, holes etc which need to be kept free of adhesive. Since such micromachined wafers cannot be coated and patterned in the usual manner, a lithographic process cannot be carried out [10]. A stamping and adhesive bonding method has been described [10], where a very thin layer of adhesive of a few microns thick is first applied to a plain wafer by spin coating. The wafer to be bonded is then pressed into the coating and separated hence transferring some adhesive to the high areas of the bond wafer. The wafer is subsequently aligned with its counterpart and the adhesive cured. The stages of this technique are illustrated in figure 2 .

Klink et al. [10] carried out investigations of wafer bonding using two different adhesives, a thermal curing two-component epoxy and a thermoplastic polymer. Using the adhesive stamping method described above a disadvantage of the epoxy adhesive was found to be the risk of contaminating the bond apparatus with cured adhesive and was also seen to heavily wet the silicon surface causing very high intrinsic attractive forces. The thermoplastic adhesives could however be used in solid form instead of wet coatings at room temperature. This allowed easier wafer handling and reduced the risk of contaminating the adhesive-free areas [10]. Furthermore, with the thermoplastic adhesive the bond process was reversible.

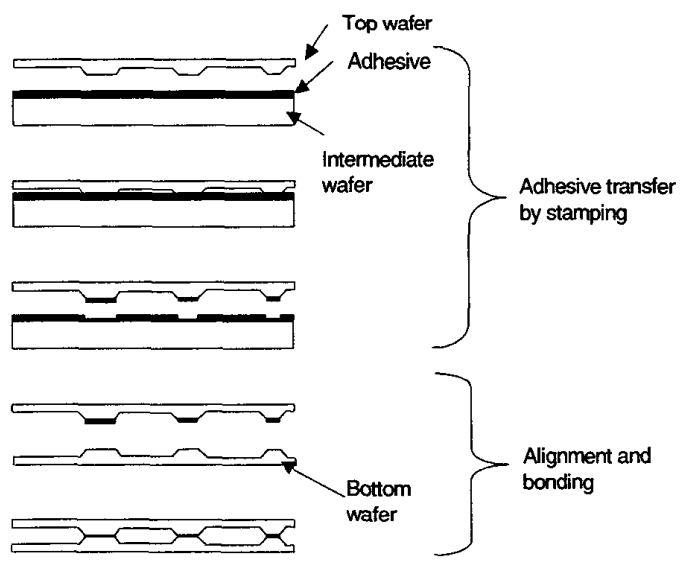

Fig. 2 Wafer bonding using the stamping technique [10]

Adhesion measurements on the bonded chips in pull tests showed the epoxy adhesives to be stronger than the thermoplastic polymers, but revealed poor bonds for smaller patterns.

The technique has been applied to the fabrication of the electrostatic actuation unit of micropumps [11] and for the sealing of an acceleration sensor. Klink et al. [10] have found satisfactory adhesion and sealing properties in the joints, but observed some limitations in the chemical stability of the thermoplastic adhesive.

\section{Wafer transfer Technique}

Although silicon is the dominant semiconductor material in MEMS due to its excellent properties, a growing number of applications require MEMS devices fabricated on other substrates [12]. Examples of this are: high power devices requiring substrates with high thermal conductivity such as diamond or silicon carbide and RF applications needing semiinsulating substrates such as sapphire [12]. Fabricating these devices monolithically on the particular substrate is not usually compatible with standard processes. Nguyen et al. [12] have developed a new wafer transfer technique by which surface micromachined layers can be removed and re-attached on a desired substrate. The full detail of the wafer transfer process is given in the paper but it involves the removal of the original substrate followed by the new substrate then being bonded to the device layers using a variety of techniques. They found Norland Optic's epoxy to provide the reliability and durability required in their particular application. The room temperature UV cured epoxy not only avoids the problems normally associated with heat, it also tolerates the harsh solvents and $\mathrm{HF}$ acid that are used in their process.

\section{Micro-gasketing for Liquid Filled MEMS Devices}

Microfluidic MEMS devices contain and/or handle small quantities of liquids in order to carry out chemical or biological analysis. Micromachining technology is used to produce passive channels in a microdevice within which the liquids can be stored or pumped around to enable mixing. The motion of the sample and reagent fluids is accomplished by the application of voltages or externally generated pressures [13]. Typically, a closed liquid reservoir in a fluidic device contains liquid volumes ranging from $\mu 1$ to $\mathrm{ml}$ and the enclosure and sealing of the liquids within the device presents many challenges.

Simon et al. [2] have reported an approach to filling and sealing of multiple microdevices with a volume less than $1 \mathrm{nl}$. The liquids are sandwiched between the silicon substrate and a glass layer, as shown in figure 3. A Teflon microgasketing technique is used in conjunction with a room temperature (as they are limited to a low temperature sealing method) UV curing epoxy adhesive to isolate the liquid inside lithographically defined areas permanently sealing the device. One of the key challenges of using adhesives for wafer joining in MEMS is how to apply the adhesives on selected areas only. Simon et al. [2] have developed a method which uses wicking 
microchannels to achieve good penetration of the adhesive between the silicon and glass cover throughout the entire die. Simon et al. [2] used this method in the construction of the liquid filled microrelays as illustrated in figure 4 where a series of Teflon lines are patterned around the edge of the die fabricated in the Teflon gasket layer. This helps in drawing the adhesive into the centre of the die using capillary action. It is found [2] that using this technique a drop of adhesive may be wicked into the die to a required position with minimal air pockets.

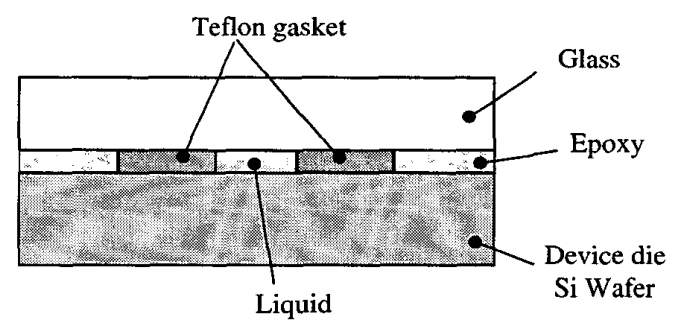

Fig. 3 Liquid filled gasketed area sealed with epoxy [2]

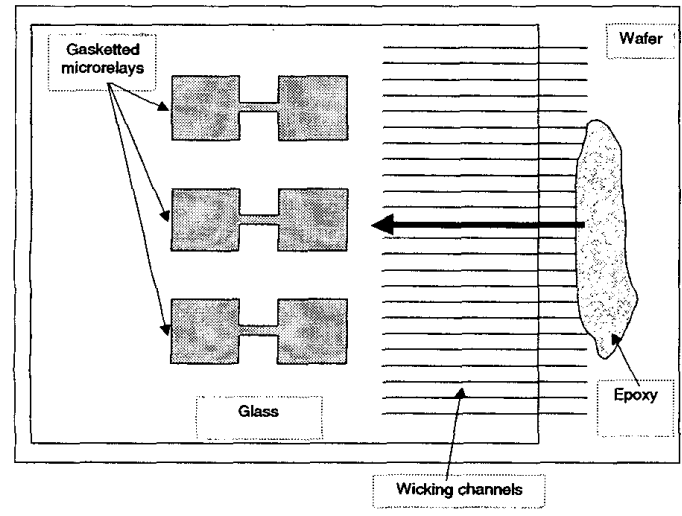

Fig. 4 Surface micromachined wicking channels [2]

\section{AdHESIVES IN MICROOPTIC ELECTROMECHANICAL SYSTEMS(MOEMS)}

There are a number of areas in MOEMS that adhesives are used. Optical cements are used to join refracting surfaces such as fibre optics and structural adhesives are used to attach together mechanical parts or to hold optical components to mechanical components. Adhesives have also been used as hydrophobic layers which are selectively removed to reveal hydrophilic areas on the underlying substrate upon which microlenses are constructed.

There are clear benefits in miniaturisation of optoelectronic components with faster processing, material costs and power requirements being amongst the more important benefits. The miniaturisation of packages however leads to more complex integration processes. One of the most challenging problems is the very precise alignment that is required for the source and waveguide. One estimate in 1998 attributed 40 to 50 percent of these component's cost to the alignment and packaging of diode lasers and the optical fibre counterparts, often requiring expensive, custom made machine vision systems and robots [14]. A well known technique used for the fibre positioning and alignment is a v-groove etched on the substrate [14-17]. Once alignment is achieved the fibre is generally fixed into the $v$-groove using an adhesive with the same refractive index [14].

\section{Optical Cement}

Where refracting surfaces are joined together, the adhesive must be transparent and homogeneous in the spectral region of interest [18]. The important properties of optical adhesives are given fully in the paper by Eberhardt. Some of the main points from the paper are given below:

Optical properties - high light transmission, colourless, matching refractive index with the interface, minimal fluorescence and homogenous.

Mechanical properties - minimal shrinkage, resistance to vibration and mechanical and thermal shock, stability over the temperature range of use, UV resistance, moisture resistance and strain-free

Chemical and Biological properties - stable, inert to interfacing materials, non-toxic

\section{Application properties - easily prepared and applied}

In optical telecommunication, laser light has to be coupled into glass fibres. This can be done by a combination of micro-lenses with dimensions of less than $1 \mathrm{~mm}$ [19]. Bonding of these lenses needs extremely small amounts of adhesive, otherwise they cannot be adjusted within the necessary small tolerances. A microdispensing technology has been developed [19] that can dispense amounts of adhesive less than 100 picolitres for bonding of micro-lenses.

\section{Microlens Manufacture}

The role of optics is becoming more and more important in today's world of information processing as the performance of communication and display technologies continue to increase. For many opticsbased applications, the low-cost fabrication of microlenses is becoming vital.

Hartmann et al. [1] have developed a low-cost method for the manufacture of polymer microlenses for use in optical systems [1]. An adhesive 
hydrophobic layer is applied to the substrate by mechanical polishing; adhesive coating of RainX has been used on $\mathrm{Si}, \mathrm{SiO} 2$, and $\mathrm{SiN}$, and an adhesive coating of Turtle Wax Super Hard Shell Car Wax on GaAs, InP, GaInAs, and other III-V materials, to make these materials hydrophobic. Hydrophilic domains are then lithographically patterned and the hydrophobic layer selectively etched away using $\mathrm{O}_{2}$ plasma. The substrate is then dipped into a UV-curable monomer solution, which will attach to the hydrophilic domain as lenses. After curing the microlenses become hard and stable.

Lenses with lower $f$-numbers can be manufactured by re-dipping the substrate into the monomer solution. The monomer assembles on top of the existing cured lenses causing an increase in the radii of curvature and a corresponding reduction in $f$-numbers. Double-sided convex lenses can also be constructed by applying the hydrophobic adhesive to both sides of a very thin transparent substrate. Photoresist is then coated on both sides and patterned using a single lithographic exposure. In this way aligned patterns are formed on the two sides of the substrate constituting the footprints of a double-convex lens.

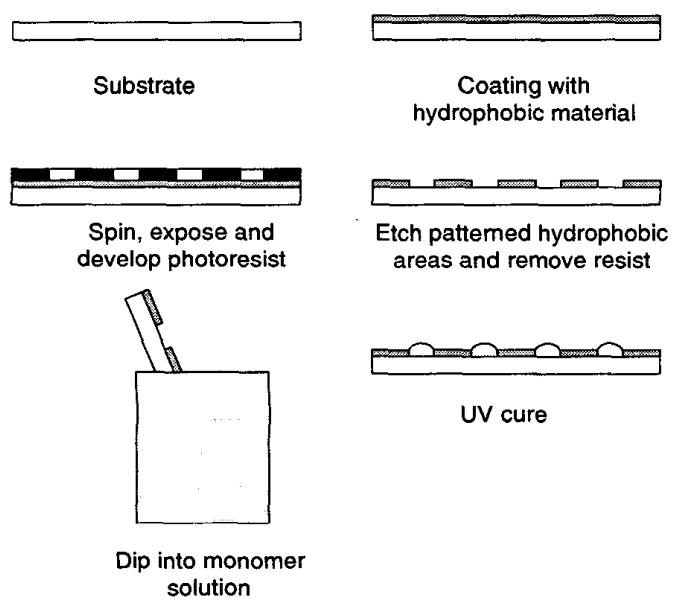

Fig. 5 Process Flow for Hydrophobic Patterning [1]

In a separate study [20] fabricated PMMA (polymethylmethacrylate) refractive microlens arrays using transparent acrylic resin. An orthodontic resin from DENTSPLY International Inc., which is commonly used in dentistry, was used. It consists of a transparent acrylic resin kit including MMA liquid and polymerisation powder. A PMMA layer was formed on the substrate through spin coating of the resin. The designed pattern of the lens structure was then transferred to the PMMA layer by Reactive Ion Etching (RIE) with an oxygen plasma. Subsequent thermal treatment caused the PMMA to reflow forming the final lens shape. Microlenses with diameters in the range of $200-500 \mu \mathrm{m}$ were produced.

\section{RELIABILITY OF ADHESIVE BONDED ASSEMBLIES}

There are a number of key failure mechanisms which impact on the reliability of MEMS devices, including stiction and wear, delamination, cyclic mechanical fatigue, loss of hermeticity and environmentally induced failures [21].

In the IC industry, the bond layer serves as the foundation and often the weak link in the reliability of the chip packages. MEMS packages are likely to have a greater number of bond layers with more stringent requirements. The additional bond layers arise from multiple surfaces inside the package, which often must maintain precise component or chip alignment.

\section{Stress}

A major drop in reliability may be caused by excess stress in the package. The stress can be the result of stress inducing fabrication processes, CTE mismatch in the die attach materials, lid sealing, or shrinkage during adhesive curing. Stresses in MEMS devices can result in deformation leading to misalignment of gear teeth, device breakage and buckling of long beam elements. Furthermore, microengineered silicon components with micronscale moving parts are typically fragile and hence sensitive to stresses. Mechanical stress can lead to inaccurate results in the case of microsensors such as piezoresistive or piezoelectric elements [21]. If stressed, the sensor will give inaccurate measurements and the device response may drift in the long-term, due to the slow accumulation of creep in the adhesive bond between the silicon die and the package housing.

One of the sources of stress in MEMS devices results from the die attach material at the interface between MEMS die and the package substrate [22]. Depending upon the die-attach material and the thermal expansion mismatch between the package and the chip, interfacial stress can develop within the MEMS package [23]. This can be partially controlled by the use of a die attach material with a relatively low modulus of elasticity. Conventional epoxy type adhesives tend to have relatively high moduli and hence are not generally as effective in minimising thermomechanical stresses compared to low moduli thermoplastic adhesives [8]. Furthermore, thermoplastic materials have the advantage that their modulus remains low in service, whereas the modulus of a typical epoxy will tend to increase [8].

Micromachined accelerometers are amongst the most widely used MEMS sensors, largely used in automotive airbag systems. In recent work [24], a packaging study was carried out on a two-chip design of accelerometer to provide an understanding and guidance for designing a package with low stress and deformation. The sensor consisted of two silicon chips 
with a surface micromachined capacitive transducer and a microprocessor control unit. The two chips were die bonded onto a single leadframe using wire bonding and moulded with an epoxy compound. Two die bonding techniques were considered, full die attach and a four-dot die-attach process. The test data indicated that the four-dot room-temperature vulcanising (RTV) silicone die-attach process resulted in a significantly lower packaging stress to the transducer which and is therefore appropriate for stress sensitive MEMS devices.

\section{Outgassing}

When adhesives such as epoxies or high performance resin cyanate esters are used, the die attach compounds outgas as they cure [22]. The organic vapours and water deposit on MEMS and MOEMS features, in crevices and on bond pads. This may lead to device stiction and/or cause corrosion. Recommended solutions include the use of very low outgassing die attach materials and the removal of outgassing vapours during the curing stage. The use of appropriate moisture particle getters have also been considered in MEMS [25-26] and MOEMS [27]. Getters are referred to as materials which chemically attract active gases or particles in a vacuum environment with the role of maintaining and improving vacuum. Their use allows high vacuum levels in a sealed MEMS package thereby prolonging their operational lifetimes [25].

\section{Delamination}

Sandborn et al. [28] carried out a study to rank the suitability of bond layer materials for chip-to-chip attachments. They tested a number of bond materials including epoxy, thermoplastic and solder. Accelerated ageing, thermal cycling and humidity tests were carried out for 100 days. In general they found solder outperformed the adhesives. The thermoplastics also performed well during ageing and temperature cycling tests, however the epoxy films were seen to experience moderate delamination growth in all tests.

\section{Hermeticity}

The key specifications established by the aerospace industry for MEMS devices are that the encapsulant should display resistance to mobile ion permeation and high humidity, and constant thermal and mechanical properties within wide temperature extremes $\left(-55^{\circ} \mathrm{C}\right.$ to $100^{\circ} \mathrm{C}$ ). Low internal stresses are also important to guarantee accuracy in microsensors. Historically, high purity epoxies have been used as reliable, nonhermetic encapsulation materials.

In a search for a high performance conformal coating for protecting MEMS devices from adverse environments as well as mechanical stresses, an investigation was carried out [5] where a number of commercial conformal encapsulation materials used for low cost aerospace applications were evaluated. These included silicone elastomers, epoxies and Parylenes. To determine the environmental protection offered by each material, a number of properties were characterised including mobile ion permeation and moisture ingress resistance, interfacial adhesion variation through thermal shock cycling and 85 ${ }^{\circ} \mathrm{C} / 85 \%$ RH ageing. The encapsulants used in their study were:

- Dexter Hysol single component thermal cure epoxy resins FP4450 and FP4460

- Dow Corning two component thermal cure silicone encapsulants Q1-4939 and DC1-4207

- Dow Corning single component RTV silicone encapulants DC1-2577, DC1-2620, DC1-3140 and DC1-3145 clear

Epoxies generally have an inherent weakness in high humidity environments. However the epoxy based FP4450 and FP4460 encapsulants were found to have excellent adhesion and fair resistance to moisture ingress making them attractive glob-top candidates. Surface Insulation Resistance (SIR) tests showed that FP4450 offered a higher degree of environmental protection than the FP4460. The silicone encapsulant Q1-4939 also provided the best environmental protection and was highly recommended for applications requiring low stress encapsulation.

\section{CONCLUSIONS}

A comprehensive review has been carried out on the use of adhesives in MEMS and MOEMS technologies as reported in the literature and the web. The study has looked in detail at the failure mechanisms and inherent properties of adhesives that impact on the reliability of MEMS devices.

It has been found that although there is an abundance of material available on MEMS technology and their manufacture, there is a very limited amount of information disseminated in the available literature in relation to the use of adhesives and in particular the type of adhesive used.

\section{ACKNOWLEDGEMENT}

This work has been performed as part of the activities of the Adhesives in MEMS and MicroOptics Working Group of the European Union funded Adhesives Network. This network has been established to promote information exchange between a number of European partners involved in electronic and microsystems manufacture. 


\section{REFERENCES}

[1] D.M. Hartmann, Osman Kibar, and Sadik C. Esener: "Characterization of a polymer microlens fabricated by use of the hydrophobic effect", Optics Letters, Vol. 25, No. 13, July 1,2000

[2] J. Simon et al.: "Microgasketing and Room Temperature Wafer Joining for Liquid-Filled MEMS Devices", ASME Joumal of Microelectromechanical Systems (MEMS), pp. 2934, 1997.

[3] Nasa Glenn Research Centre, http://www.grc.nasa.gov/WWW/MED/skills/mems/project.ht $\mathrm{m}$

[4] Aeroprobe Corporation: "MEMS-Based, High Frequency Response, 5-Sensor Probe", http://www.aeroprobe.com/mems_probe.htmL

[5] J. Wu et al.: "Evaluation and Characterisation of Reliable Non-Hermetic Conformal Coatings for Microelectromechanical System(MEMS) Device Encapsulation", IEEE Trans. On Advances Packaging, Vol. 23, No. 4, November 2000.

[6] Thermoset Advanced Electronic Materials, Datasheet MD-140

[7] Dexter Electronic Materials, Datasheet QMI 5030.

[8] P. M. Bartholomew: "Review of Conductive Adhesives and Ink for Microengineered Devices", IEE Colloquium on Assemly and Connections in Microsystems, February 1997, London.

[9] M. K. Heschel et al.: "Stacking Tecknology for a Space Constrained Microsystem", Proc. 11th Int. Workshop on MicroElectroMechanical Systems(MEMS 98), pp. 312-317, NY, 1998.

[10] G. Klink and B. Hilerich: "Wafer bonding with an Adhesive Coating", Proc. SPIE conf. On Micromachined Devices and Components IV, 1998, pp. 50-61

[11] R. Linnemann et al.: "Full-wafer-mounting Technologies for Silicon Micropumps", Proc. Eurosensors XI, Warsaw, 1997.

[12] H. Nguyen et al.: "A Substrate-Independent Wafer Transfer Technique for Surface-Micromachined Devices", The 13th IEEE Int. Micro Electro Mechanical Syatems Conf.(MEMS 2000), Jan. 23- 27, 2000, Miyazaki, Japan.

[13] D. J. Nagel and M. E. Zaghloul: "MEMS: Micro Technology, Mega Impact", Circuits and Devices, pp. 14 - 25, March 2001.

[14] R. W. Hardin: "MEMS has Benefits for Single-mode Fibre Alignment and Lasers", SPIE OE Reports, 176, August 1998.

[15] M.A. Chan et al.: "A Micromachined Pressure Sensor with Fiberoptic Interferometric Readout", Sensors and Actuators A-Physical, 43 (1-3), pp.196-201, May 1994.
[16] Y. Sunaga et al.: "2 Gbit/s small form factor fiber-optic transceiver for single mode optical fibre", IEEE Transaction on Advanced Packaging, 23 (2), pp.176-181, MAY 2000.

[17] J-P Gouy et al.: "3-D Microsystem Packaging for Interconnecting Electrical, Optical and Mechanical Microdevices to the External World", http://www.fujita3.iis.utokyo.ac.jp/ limms/projects/JPGproj.htm.

[18] R. Eberhardt: "State of the Art and Future Requirements for Optical Transparent Adhesives in Microoptics and Optoelectronics", Proc. 2000 Workshop on Polymeric Materials for Microelectronics and Photonics Applications: Mechanics, Physics, Reliability, Processing, London, UK, Dec 4-5, 2000.

[19] W. Meyer: "Microdispensing of Adhesives and Other Polymers", Polytronics 2001, 1st International IEEE Conference on Polymers and Adhesives in Microelectronics and Photonics, Potsdam, Germany, http://www.microdrop.de/

[20] S-H Ahn, et al.: "Fabrication of PIMMA Refractive Microlens Array Using Transparent Acrylic Resin", 3rd Micro Opt Electro Mechanical Systems (MOEMS) Conf., USA, 1999. http://plaza.snu.ac.kr/ micro/research/lens.htm

[21] R. R. Tummala: Fundamentals of Microsystems Packaging, McGraw-Hill, New York, 2001.

[22] C. B. O'Neal, et al.: "Challenges in the Packaging of MEMS", International Symposium on Advanced Packaging Materials, pp. $41-47,1999$.

[23] M. L. Kniffin et al.: "Packaging for Silicon Micromachined Accelerometer", The International Journal of Microcircuits and Electronic Packaging, Vol. 19, No. 1, pp. 75-86, 1996.

[24] G. Li and A. A. Tseng: "Low Stress Packaging of a Micromachined Accelerometer", EEE Trans. On Electronics Packaging Manufacturing, Vol. 24, No1, January 2001, pp. 18-25.

[25] P. W. Bames: "Sealing MEMS Devices in Ceramic Packages in a High Vacuum Atmosphere", Technical Bulletin, SST International, www.sst-tech.com/FDF/sealmems.pdf.

[26] R. Ramesham: "Nonevaporable Getters To Maintain Vacuum in Sealed MEMS", http://www.nasatech.com/Briefs/Feb00/NPO20617.html. 2000.

[27] K. Gilleo and S. Corbett: "Getters - Molecular Scavengers for Packaging", HDI, pp. 26 - 29, Jaruary 2001.

[28] P. Sandbom et al.: "Test and Evaluation of Chip-to-Chip Attachment of MEMS Devices, Proc. ITHERM 2000, Las Vegas, Nevada, USA, pp. 133-140, May 23-26 2000. 